\title{
A new precise measurement of g-2 of muon
}

\author{
Ivan Logashenko* \\ Department of Physics, Boston University, Boston, MA 02215, USA \\ Budker Institute of Nuclear Physics, Novosibirsk, 630090, Russia \\ E-mail: 'Iogashenko@inp.nsk.su'
}

H.N. Brown ${ }^{2}$, G. Bunce ${ }^{2}$, R.M. Carey ${ }^{1}$, P. Cushman ${ }^{9}$, G.T. Danby ${ }^{2}$, P.T. Debevec ${ }^{7}$, M. Deile ${ }^{12}$, H. Deng ${ }^{12}$, S.K. Dhawan ${ }^{12}$, V.P. Druzhinin ${ }^{3}$, L. Duong ${ }^{9}$, E. Efstathiadis ${ }^{1}$, F.J.M. Farley ${ }^{12}$, G.V. Fedotovich ${ }^{3}$, S. Giron ${ }^{9}$, F. Gray ${ }^{7}$, D. Grigoriev ${ }^{3}$,

M. Grosse-Perdekamp ${ }^{10}$, A. Grossmann ${ }^{6}$, M.F. Hare ${ }^{1}$, D.W. Hertzog ${ }^{7}$, V.W. Hughes ${ }^{12}$, M. Iwasaki ${ }^{11}$, K. Jungmann ${ }^{6}$, D. Kawall ${ }^{12}$, M. Kawamura ${ }^{11}$, B.I. Khazin ${ }^{3}$, J. Kindem ${ }^{9}$, F. Krienen ${ }^{1}$, I. Kronkvist ${ }^{9}$, R. Larsen ${ }^{2}$, Y.Y. Lee ${ }^{2}$, R. McNabb ${ }^{9}$, W. Meng ${ }^{2}$, J. Mi ${ }^{2}$, J.P. Miller ${ }^{1}$, W.M. Morse ${ }^{2}$, D. Nikas ${ }^{2}$, C.J.G. Onderwater ${ }^{7}$, Y. Orlov ${ }^{4}$, C. Özben ${ }^{2}$, J.M. Paley ${ }^{1}$, C. Polly ${ }^{7}$, J. Pretz ${ }^{12}$, R. Prigl ${ }^{2}$, G. zu Putlitz ${ }^{6}$, S.I. Redin ${ }^{12}$, O. Rind ${ }^{1}$, B.L. Roberts ${ }^{1}$, N. Ryskulov ${ }^{3}$, Y.K. Semertzidis ${ }^{2}$, Yu.M. Shatunov ${ }^{3}$, E. Sichtermann ${ }^{12}$, E. Solodov ${ }^{3}$, M. Sossong ${ }^{7}$, A. Steinmetz ${ }^{12}$, L.R. Sulak ${ }^{1}$, C. Timmermans ${ }^{9}$, A. Trofimov ${ }^{1}$, D. Urner ${ }^{7}$, P. von Walter $^{6}$, D. Warburton ${ }^{2}$, D. Winn ${ }^{5}$, A. Yamamoto ${ }^{8}$, D. Zimmerman ${ }^{9} \dagger$ MUON G-2 COLLABORATION

Abstract: The Muon (g-2) Experiment (E821) at Brookhaven National Laboratory (BNL) started data taking in 1997. The latest result $a_{\mu^{+}}=11659202(14)(6) \times 10^{-10}$, based on statistics of about 1 billion decays collected in 1999, is in good agreement with the previous measurement. With the precision of $1.3 \mathrm{ppm}$, the result, compared with the Standard Model evaluation $a_{\mu}(S M)=11659159.6(6.7) \times 10^{-10}$, shows a difference of $a_{\mu}(\exp )-a_{\mu}(S M)=43(16) \times 10^{-10}$.

\footnotetext{
${ }^{*}$ Speaker.

${ }^{\dagger 1}$ Department of Physics, Boston University, Boston, MA 02215, USA; ${ }^{2}$ Brookhaven National Laboratory, Upton, NY 11973, USA; ${ }^{3}$ Budker Institute of Nuclear Physics, Novosibirsk, Russia; ${ }^{4}$ Newman Laboratory, Cornell University, Ithaca, NY 14853, USA; ${ }^{5}$ Fairfield University, Fairfield, CT 06430, USA; ${ }^{6}$ Physikalisches Institut der Universität Heidelberg, 69120 Heidelberg, Germany; ${ }^{7}$ Department of Physics, University of Illinois at Urbana-Champaign, IL 61801, USA; ${ }^{8}$ KEK, High Energy Accelerator Research Organization, Tsukuba, Ibaraki 305-0801, Japan; ${ }^{9}$ Department of Physics, University of Minnesota, Minneapolis, MN 55455, USA; ${ }^{10}$ RIKEN-BNL Research Center; ${ }^{11}$ Tokyo Institute of Technology, Tokyo, Japan; ${ }^{12}$ Department of Physics, Yale University, New Haven, CT 06511, USA
} 


\section{Introduction}

The magnetic moment of a particle is related to its spin as $\vec{\mu}=g(e / 2 m c) \vec{S}$. According to Dirac's theory, the gyromagnetic factor $g$ is equal to 2 for point-like particles, such and electron or muon. In the quantum fied theory it slightly deviates from $2, g=2(1+a)$, where $a$ is the anomalous magnetic moment. All existing fundamental interactions contribute to the anomaly, with different strength.

The electron's anomalous magnetic moment is the most precisely measured with an accuracy of 4 parts per billion (ppb) 陑. Even at this level, only the QED contribution is significant. As a result, $a_{e}$ currently provides the best determination of the fine structure constant, under the assumption of the validity of QED.

The muon's anomalous magnetic moment is measured with an accuracy of $1.3 \mathrm{ppm}[2]$ in the experiment described by this paper. Compared to the electron, the muon $g$ value is more sensitive to non-QED contributions, typically by a factor of $\left(m_{\mu} / m_{e}\right)^{2}$. Therefore, although measured about 350 times less precisely than the electron, the muon anomaly is much more sensitive to the physics beyond the standard model.

\section{Experimental setup}

The experimental principle in the Brookhaven experiment is similar to that of the final

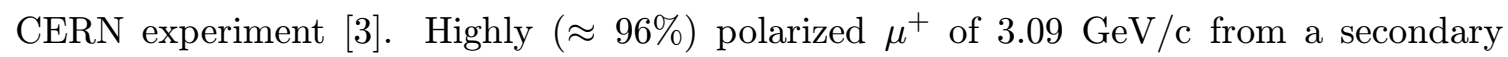
beamline are injected through a superconducting inflector into a storage ring $14.2 \mathrm{~m}$ in diameter with an effective circular aperture of $9 \mathrm{~cm}$ in diameter. A pulsed magnetic kicker gives a $10 \mathrm{mrad}$ deflection which places muons onto stored orbits. The superferric storage ring [ien has a homogeneous magnetic field of $1.45 \mathrm{~T}$. In the lab frame, the spin of the stored muons precess relative to the momentum vector with the angular frequency

$$
\overrightarrow{\omega_{a}}=-\frac{e}{m_{\mu}}\left[a_{\mu} \vec{B}-\left(a_{\mu}-\frac{1}{\gamma^{2}-1}\right) \vec{\beta} \times \vec{E}\right] .
$$

At the "magic" momentum of muons $p_{\mu}=3.09 \mathrm{GeV} / \mathrm{c}(\gamma=29.3)$, the second term cancels out. As a result, the precession frequency does not depend on the electric field, which allows us to use the electrostatic quadrupole field for vertical beam focusing.

The precession frequency $\omega_{a}$ and the magnetic field $B$ are measured simultaneously, and then $a_{\mu}$ is calculated according to $\left(\overline{2} \overline{2}_{1} \overline{1}_{1}\right)$. Since the precession frequency is proportional to $a_{\mu}$ itself, not $g$, such an approach allows to achieve higher precision via the direct measurement of the anomaly. The magnetic field is determined using the NMR technique that measures the free proton precession frequency $\omega_{p}$ in the same magnetic field seen by the muons. The anomaly is then extracted through the relation

$$
a_{\mu}=\frac{\omega_{a} / \omega_{p}}{\mu_{\mu} / \mu_{p}-\omega_{a} / \omega_{p}}
$$

where the only external input is the ratio of muon to proton magnetic moments, $\mu_{\mu} / \mu_{p}=$ $3.18334539(10)$ [ [in: 
analyses, one of the field and one of the spin precession frequency. Experimenter bias is eliminated by maintaining secret offsets between the two analysis groups. Once both analyses are complete, the results are frozen, the offsets are revealed and only then is the value of $a_{\mu}$ determined.

\section{Measurement of the magnetic field}

The magnetic field is measured with various systems of NMR probes. Inside the storage region, seventeen NMR probes are used, mounted in an array on a trolley, which moves on a fixed track inside the muon storage ring vacuum. Typically, the trolley measurements are done every 3 4 days during the data taking periods. Interpolation of the field in the periods between the trolley measurements is based on the readings of about 150 fixed NMR probes, distributed around the ring in the top and bottom walls of the vacuum cham-

\begin{tabular}{|l|c|}
\hline Source of errors & Size $[\mathrm{ppm}]$ \\
\hline Absolute calibration of standard & 0.05 \\
probe & \\
Calibration of trolley probes & 0.20 \\
Trolley measurements of $B_{0}$ & 0.10 \\
Interpolation with fixed probes & 0.15 \\
Inflector fringe field & 0.20 \\
Uncertainty from muon distribution & 0.12 \\
Others $\dagger$ & 0.15 \\
\hline Total systematic error on $\omega_{p}$ & 0.4 \\
\hline$\dagger$ higher multipoles, trolley temperature and its \\
power supply voltage response, and eddy currents \\
from the kicker.
\end{tabular}

Table 1: Systematic errors for the $\omega_{p}$ analysis ber. In addition, the average reading of a subset of 36 uniformly distributed fixed probes is maintained to $0.1 \mathrm{ppm}$ by feedback to the main magnet power supply. Before and after the data taking periods, the trolley

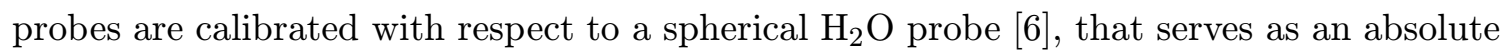
reference, to an accuracy of $0.2 \mathrm{ppm}$.

The magnetic field $B$ in $\left(\overline{2} \cdot \bar{l}_{i}^{\prime}\right)$ is the average magnetic field seen by stored muons. As was shown with the tracking of 4000 simulated muons, it agrees to $0.05 \mathrm{ppm}$ with the field $\langle B\rangle$, taken at the beam center and averaged over the azimuth. The latter was used for the $a_{\mu}$ measurement.

Two largely independent analyses of the magnetic field data were made, using different selections of NMR probes. Their results agree to within $0.03 \mathrm{ppm}$. The final value for the average magnetic field (expressed in the terms of NMR frequency of the free proton) is $\omega_{p} / 2 \pi=61791256 \pm 25 \mathrm{~Hz}(0.4 \mathrm{ppm})$, with the list of the contributing systematic errors shown in Table

\section{Measurement of the spin precession frequency}

The spin precession frequency is obtained from the muon decay time spectrum. In the muon rest frame, the parity violating nature of the weak decay $\mu^{+} \rightarrow e^{+} \nu_{e} \bar{\nu}_{\mu}$ causes the positrons to be emitted preferentially along the muon spin direction. When boosted into the lab frame, this results in a strong correlation between the positron energy and the angle 
between the muon spin and momentum vectors. The count rate of positrons observed with energy above $E_{t}$ is modulated by the muon spin precession as

$$
N(t)=N_{0}\left(E_{t}\right) e^{-t / \gamma \tau}\left[1+A\left(E_{t}\right) \cos \left(\omega_{a} t+\phi_{a}\left(E_{t}\right)\right)\right],
$$

where $\gamma \tau \approx 64.4 \mu \mathrm{s}$ is the dilated muon lifetime.

The decay positrons are detected by 24 lead/scintillating fiber calorimeters placed symmetrically along the inner wall of the vacuum chamber. The photomultiplier signals, with typical FWHM of $5 \mathrm{~ns}$, are sampled every $2.5 \mathrm{~ns}$ by a 8-bit waveform digitizers (WFD). The time and the energy of the positron are extracted from the fit of the corresponding WFD trace to an average pulse shape, measured for each calorimeter. Two completely independent software packages, implementing slightly different versions of the pulse reconstruction algorithm, were developed and the whole data set was processed with each one.

The observed spectrum is distorted by the apparatus and, therefore, is slightly different from the ideal one, described by $\left(\overline{4} \overline{4} \cdot \overline{1}_{1}\right)$. The additional effects, including pileup, coherent betatron oscillations, muon losses, bunched beam, gain shifts, were either taken into account by modifying the fitting function, or were eliminated from the data by modifying the time spectrum. All effects combined change an early part of the time spectrum by about $1 \%$.

Four independent $\omega_{a}$ analyses were made, based on two independent data productions. Three analyses were based on the fit of time spectrum itself with different treatments of pileup, gain corrections and muon losses. In the fourth analysis, the initial time spectrum is modified so that it could be fit with a function of only 3 parameters: $\omega_{a}$, $\phi_{a}$ and $A$. In such an approach some of the effects are suppressed. All four analyses produced internally-consistent results and produced an acceptable $\chi^{2}$.

The results from the four analyses

\begin{tabular}{|l|c|}
\hline Source of errors & Size $[\mathrm{ppm}]$ \\
\hline Pileup & 0.13 \\
AGS background & 0.10 \\
Lost muons & 0.10 \\
Timing shifts & 0.10 \\
E field and vertical betatron os- & 0.08 \\
cillation & \\
Binning and fitting procedure & 0.07 \\
Coherent betatron oscillation & 0.05 \\
Bunched beam & 0.04 \\
Gain changes & 0.02 \\
\hline Total systematic error on $\omega_{a}$ & 0.3 \\
\hline
\end{tabular}

Table 2: Systematic errors for the $\omega_{a}$ analysis were found to agree within $0.3 \mathrm{ppm}$, consistent with statistical variation of $0.4 \mathrm{ppm}$, expected from the use of slightly different subsets of data. The value of $\omega_{a}$ obtained from the fit was corrected by $+0.81 \pm 0.08 \mathrm{ppm}$

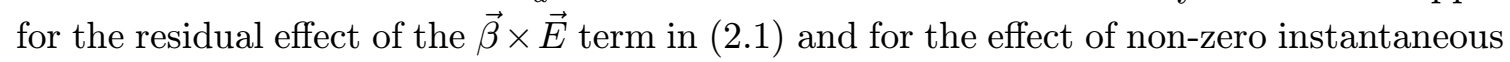
angle between the spin and momentum vector due to vertical betatron oscillations. The final value for the muon spin precession frequency is $\omega_{a} / 2 \pi=229072.8 \pm 0.3 \mathrm{~Hz}(1.3 \mathrm{ppm})$, with the list of the contributing systematic errors shown in Table ${ }_{2}^{2}$

Using the measured values of the $\omega_{p}$ and $\omega_{a}$, the value of the anomalous magnetic moment of muon was derived following (2, $(\overline{2} \cdot \overline{2})$ :

$$
a_{\mu^{+}}=11659202(14)(6) \times 10^{-10}(1.3 \mathrm{ppm})
$$




\section{Results and discussion}

The new result dominates the new world average $a_{\mu}(\exp )=11659203(15) \times 10^{-10}(1.3 \mathrm{ppm})$, obtained as the weighted mean of the new and all previous measurements. The difference between the $a_{\mu}(\exp )$ and the theoretical value from the standard model $a_{\mu}(\mathrm{SM})$ is

$$
a_{\mu}(\exp )-a_{\mu}(\mathrm{SM})=43(16) \times 10^{-10} .
$$

The standard model calculation [i] takes into account the contributions from the quantum electrodynamics $a_{\mu}(\mathrm{QED})=11658470.56(0.29) \times 10^{-10}(0.025 \mathrm{ppm})$, the weak $a_{\mu}($ weak $)=$ $15.1(0.4) \times 10^{-10}(0.03 \mathrm{ppm})$ and the strong interactions $a_{\mu}(\mathrm{had})=673.9(6.7) \times 10^{-10}(0.57$ $\mathrm{ppm})$. Independent theoretical evaluations performed after publication of the new $a_{\mu}(\exp )$ value, generally agree with $\left[\overline{\bar{q}_{1}}\right]$. A lot of effort has been put into improving the evaluation of the hadronic contribution $a_{\mu}(\mathrm{had})$, which dominates the uncertainty of the theoretical value. Further significant improvement is expected when new data on $e^{+} e^{-} \rightarrow$ hadrons from Novosibirsk, Beijing and ongoing experiments at DAPHNE and B-factories will be included in the calculations. The topic is reviewed in a number of recent publications

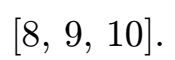

In 2000, approximately 4 times more data were collected than in 1999. Analysis of that data is in progress, and a factor of two improvement in the precision of $a_{\mu}(\exp )$ is expected. In 2001, another three times the data volume compared to 1999 was collected, this time with negative muons. New developments from both the experimental and theoretical sides may soon show whether the current discrepancy $(\bar{s} . \overline{1})$ is a sign of physics beyond the standard model.

\section{Acknowledgements}

This work is supported in part by the U.S. Department of Energy, the U.S. National Science Foundation, the German Bundesminister für Bildung und Forschung, the Russian Ministry of Science, and the U.S.-Japan Agreement in High Energy Physics.

\section{References}

[1] R.S. Van Dyck Jr., P.B. Schwinberg, H.G. Dehmelt, Phys. Rev. Lett. 599 (1987) $26{ }^{\prime}$

[2] H.N. Brown, et al., iPhys. Rev. Lett. 86 (2001) $222 \overline{7}$.

[3] J. Bailey, et al., 'Nucl. Phys. B $150(1979)$ 1'

[4] G.T. Danby, et al., 'Nucl. Instrum. Meth. A 457 (2001) 151.

[5] P.J. Mohr and B.N. Taylor, iRev. Mod. Phys. $\mathbf{7} \mathbf{2}(2000)$ 351.'.

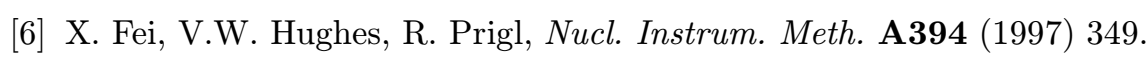

[7] M. Davier and A. Höcker, Phys. Lett. B $\mathbf{4} 35 \overline{3}$ (1998) $42 \overline{7}$.

[8] W. J. Marciano and B. L. Roberts, hep-ph/0105056.

[9] K. Melnikov, SLAC-PUB-8844, hep-ph/0105267.

[10] J.P. Miller, Proc. of XX International Symposium on Lepton and Photon Interactions at High Energies, July 23-28, 2001, Rome, Italy. 